\title{
Completude das fichas de ocorrência das unidades de suporte avançado do SAMU
}

\author{
Completeness of occurrence cards of SAMU advanced support units
}

Integridad de las tarjetas de ocurrencia de las unidades de soporte avanzado SAMU

\begin{abstract}
RESUMO
Objetivo: analisar a completude das fichas de atendimento das Unidades de Suporte Avançado do SAMU Regional Metropolitano/PR. Método: estudo documental, retrospectivo, quantitativo, realizado com 385 fichas de notificação do serviço de atendimento móvel de urgência. A coleta ocorreu entre março e agosto de 2020, com dados de agosto de 2018 à julho de 2019. A completude das fichas de ocorrência foi mensurada pela proporção de campos preenchidos. Resultados: a completude dos dados foi excelente para 10 variáveis, estas referente a identificação de chamado e paciente $(100 \%)$, clínicas $(6,7 \%)$ e de desfecho (14,3\%); muito ruim nos campos de identificação demográfica; predominantes ruim e muito ruim para as variáveis clínicas. Conclusão: evidencia-se falha do preenchimento das fichas de ocorrência, independente da localidade do chamado. Destaca-se a importância de desenvolver mecanismos para estabelecer o preenchimento completo, seja por capacitação ou desenvolvimentos de novas tecnologias para coleta de dados.
\end{abstract}

DESCRITORES: Unidades Móveis de Urgência; Enfermagem em Emergência; Serviços médicos de emergência; Registros.

\section{ABSTRACT}

Objective: to analyze the completeness of the attendance files of the Advanced Support Units of the SAMU Regional Metropolitano / PR. Method: documentary, retrospective, quantitative study, carried out with 385 notification forms from the mobile emergency care service. The collection took place between March and August 2020, with data from August 2018 to July 2019. The completeness of the occurrence records was measured by the proportion of fields filled. Results: the completeness of the data was excellent for 10 variables, these referring to the identification of the call and patient (100\%), clinical $(6.7 \%)$ and outcome $(14.3 \%)$; very bad in the fields of demographic identification; predominantly bad and very bad for clinical variables. Conclusion: there is a failure to fill out the occurrence forms, regardless of the location of the call. It highlights the importance of developing mechanisms to establish complete completion, either through training or developments in new technologies for data collection. DESCRIPTORS: Ambulances; Emergency Nursing; Emergency Medical Services; Records.

\section{RESUMEN}

Objetivo: analizar la integridad de los archivos de asistencia de las Unidades de Soporte Avanzado del SAMU Regional Metropolitano/PR. Método: estudio documental, retrospectivo, cuantitativo, realizado con 385 formularios de notificación del servicio móvil de urgencias. La recolección se llevó a cabo entre marzo y agosto de 2020, con datos de agosto de 2018 a julio de 2019. La integridad de los registros de ocurrencia se midió por la proporción de campos llenados. Resultados: la completitud de los datos fue excelente para 10 variables, estas referidas a la identificación de la llamada y paciente $(100 \%)$, clínica $(6,7 \%)$ y resultado (14,3\%); muy malo en el campo de la identificación demográfica; predominantemente malo y muy malo para las variables clínicas. Conclusión: hay una falla al completar los formularios de ocurrencia, independientemente de la ubicación de la llamada. Destaca la importancia de desarrollar mecanismos para establecer el llenado completo, ya sea mediante capacitación o desarrollos en nuevas tecnologías para la recolección de datos.

DESCRIPTORES: Ambulancias; Enfermería de Urgencia; Servicios Médicos de Urgencia; Registros.

RECEBIDO EM: 05/04/2021 APROVADO EM: 10/05/2021

\section{Jhennefer Gomes Ferreira}

Enfermeira. Graduada pelo Centro Universitário Santa Cruz - UniSantaCruz.

ORCID: 0000-0002-6961-2553 


\section{Isabeli Emily Chevonik}

Enfermeira. Especialista em Urgência e Emergência pela Secretaria Municipal de Saúde de Curitiba - SMS/Curitiba. ORCID: 0000-0002-6449-8747

\section{Josemar Batista}

Enfermeiro. Doutorando em Enfermagem pela Universidade Federal do Paraná. Professor Adjunto e Coordenador do curso de Enfermagem do Centro Universitário Santa Cruz de Curitiba - UniSantaCruz.

ORCID: 0000-0001-9838-1232

\section{Bruna Eloise Lenhani}

Enfermeira. Doutora em Enfermagem pela Universidade Federal do Paraná. Professora Adjunta do curso de Enfermagem do Centro Universitário Santa Cruz de Curitiba - UniSantaCruz.

ORCID: 0000-0002-6009-3400

\section{Larissa Marcondes}

Enfermeira. Doutoranda em Enfermagem pela Universidade Federal do Paraná. Professora Adjunta do curso de Enfermagem do Centro Universitário Santa Cruz de Curitiba - UniSantaCruz.

ORCID: 0000-0002-8745-6486

\section{INTRODUÇÃO}

$\mathbf{P}$ ercebeu-se, ao longo do tempo, que vidas poderiam ser salvas se fossem rapidamente atendidas por pessoas treinadas e qualificadas, ainda no ambiente fora dos hospitais, e transportadas a um local onde pudessem receber atendimento com suporte mais específico para cada caso. ${ }^{1}$

O cenário da saúde está em constante mudança e alguns fatores são determinantes para as entidades que ofertam serviços de saúde. Novos traços epidemiológicos populacionais, aperfeiçoamento da medicina diagnóstica, tratamentos de alta complexidade, crescimento da indústria farmacêutica, multiplicidade de convênios de saúde e políticas nacionais em saúde são alguns fatores que estão relacionados ao cenário atual e mantêm relação estreita com a qualidade dos serviços prestados. ${ }^{2}$

A qualidade dos serviços de enfermagem inclui não só a formação do enfermeiro, os registros são elementos imprescindíveis ao processo do cuidar. Quando redigidos de maneira que retrate a realidade a ser documentada, possibilitam a comunicação entre a equipe de saúde e servem a diversas outras finalidades, tais como ensino, pesquisas, auditorias, processos jurídicos, planejamento, fins estatísticos e o processo de restauração da saúde do cliente ou, quando isto não é possível, a melhor das condições de vida, as orien- tações quando ao autocuidado, a simplificação e à segurança nos procedimentos. Como os registros no prontuário do paciente são um documento legal de defesa dos profissionais, devem estar imbuídos de autenticidade e significado legal de acordo com a Resolução no0514/2016 do Conselho Federal de Enfermagem. ${ }^{3}$

Então, a qualidade do registro das ações assistenciais reflete tanto a qualidade da assistência como a produtividade do seu trabalho. Não é permitido escrever a lápis ou utilizar corretivos líquidos; não devem conter rasuras, entrelinhas, linhas em branco ou espaço. ${ }^{4}$

No Atendimento Pré-Hospitalar (APH) móvel de urgência, esses registros não são realizados no momento da prestação do cuidado, mas após a estabilização do paciente, no entanto ainda durante o atendimento. As anotações são feitas na ausência do paciente, o que requer empenho do profissional no resgaste e sistematização das informações. Diante desta especificidade, surgem dificuldades no registro dos atendimentos realizados no Serviço de Atendimento Móvel de Urgência (SAMU). As dificuldades encontradas tem relação com a falta de informações dos pacientes, por exemplo: identificação, antecedentes patológicos, hábitos de vida; ausência de padronização das anotações; registros ilegíveis e ausência de identificação profissional. ${ }^{5}$
As áreas da saúde necessitam de informação para tomada de decisão $0^{6}$, com isso a completude dos dados em prontuários ou fichas de informações têm se demonstrado área de interesse crescente para avaliar a qualidade das informações transmitida entre os profissionais. ${ }^{7}$

Diante do exposto, surge como questionamento, qual a porcentagem da completude dos registros das fichas de atendimento das ambulâncias de suporte avançado de vida do SAMU Curitiba e região metropolitana? E tem como objetivo, identificar a completude das fichas de atendimento das Unidades de Suporte Avançado do SAMU Regional Metropolitano do Paraná.

\section{MÉTODO}

Trata-se de um estudo documental, retrospectivo, de abordagem quantitativa, realizado com dados obtidos através das fichas de atendimento pré-hospitalar das Unidades de Suporte Avançado do SAMU Regional Metropolitano/PR.

A pesquisa foi realizada nos municípios que possuem as Unidades de Suporte Avançado e que fazem parte do SAMU Regional Metropolitano de Curitiba, Paraná, Brasil. Sendo eles, Curitiba, Pinhais, Almirante Tamandaré, São José dos Pinhais, Araucária e Campo Largo, totalizando 14 ambulâncias. A coleta de dados realizou-se nas de- 


\section{artigo}

Ferreira, J.G.; Chevonik, I.E.; Batista, J.; Lenhani, B.R.; Marcondes, L.;

Completude das fichas de ocorrência das unidades de suporte avançado do SAMU

pendências de cada um destes locais, onde as fichas de atendimento são armazenadas, de março a agosto de 2020.

Foram incluídas as fichas de ocorrências atendidas pelo Suporte Avançando de Vida do SAMU Metropolitano/PR, preenchidas pelos profissionais de saúde durante $o$ atendimento no período de um de agosto de 2018 à 31 de julho de 2019. Foram excluídas as fichas danificadas. O estabelecimento da temporalidade das buscas foi referente ao período do último ano com registros completos acessíveis. Para o cálculo do tamanho da amostra foi considerado o número de atendimentos mensais das Unidades de Suporte Avançado do SAMU no período de 01 de agosto de 2018 à 31 de julho de 2019 (30.951 atendimentos). Para esta pesquisa foi utilizado uma amostra estratificada a qual foi calculada usando o número total de atendimentos, com $95 \%$ de confiança e margem de erro igual a 5\%. Após a seleção das fichas por cálculo amostral, a amostra foi composta por 385 registros, que foram escolhidas conforme sorteio aleatório.

As variáveis coletadas presente na ficha de ocorrência de atendimento são referente a identificação do chamado (município, data, horário, unidade de suporte avançado (USA) acionada, base), identificação do paciente (sexo, idade) demográfica (bairro, local de ocorrência), clínicas (objetivo do chamado, antecedentes, cirurgias realizadas, medicações, principais sintomas, exame clínico, início dos sintomas, sinais vitais, escala de Glasgow, escala de Trauma, vias aéreas, respiração, ausculta pulmonar, expansividade, achados, circulação, edema, perfusão, característica do pulso, ausculta cardíaca, eletrocardiograma (ECG), exame neurológico, exame físico do pescoço, exame físico urogenital, exame físico abdômen, gineco/obstétrico), desfechos (diagnóstico, procedimentos realizados, terapêutica, evolução, encaminhamento, óbito, posição de transporte).

A completude dos dados das fichas de ocorrência foi mensurada pela proporção de campos preenchidos corretamente sem informação ignorada, em um determinado campo. Os valores foram adaptados ${ }^{8}$, sendo consideradas como excelente as informações acima de 95\%; Bom $\geq 90$ a 95\%; Regular $\geq 70$ a $90 \%$; Ruim $\geq 50$ a $70 \%$ e Muito ruim $\leq$ abaixo de $50 \%$. Os dados foram tabulados e codificados no programa Microsoft Office Excel ${ }^{\circ} 2010$, utilizando-se de procedimentos de análise estatística descritiva com os resultados expressos em frequência simples e absoluta (\%).

Respeitando os aspectos éticos a pesquisa foi submetida à apreciação do Comitê de Ética em Pesquisa da Secretaria de Saúde de Curitiba- PR, para a aprovação dos aspectos éticos e viabilidade da pesquisa com seres humanos, sendo cadastrado com o CAAE: 26483119.2.0000.0101 e aprovado com o número do parecer: 3.764.811.

\section{RESULTADOS}

Foram analisadas 385 fichas de atendimento pré-hospitalar, sendo 258 das bases de unidade avançada do município de Curitiba, 39 de São José dos Pinhais, 23 de Campo Largo, 28 de Pinhais, 19 de Almirante Tamandaré e 18 de Araucária.

A completude do preenchimento das variáveis de identificação do chamado nas fichas de ocorrência de atendimento, sendo cinco variáveis, foram excelente se considerar todos os serviços, porém se estratificar o preenchimento por município de chamado, destaca-se que Curitiba teve um preenchimento de horário bom (90,7\%), Araucária apresentou um preenchimento bom para base $(94,4 \%)$ e Pinhais teve um preenchimento regular para base de atendimento $(89,3 \%)$, conforme apresentado na Tabela 1.

Segundo a identificação do paciente, quando considerado todos os municípios a completude é excelente, contudo, destaca-se uma completude regular para o preenchimento de idade e sexo no município de Almirante Tamandaré (89,5\%) e regular somente para sexo no município de Araucária (88,9\%) (Tabela 1 ).

Para identificação demográfica (bairro e local de ocorrência), todos os municípios tiveram uma completude muito ruim, destacando-se para Araucária que não preencheu nenhuma das fichas de ocorrência a variável bairro (Tabela 1 ).

Tabela 1. Completude dos campos de preenchimento das fichas de ocorrência de atendimento das Unidades de Suporte Avançado do SAMU Regional Metropolitano/PR das variáveis de identificação de chamado, do paciente e demográficas. Curitiba, Paraná, Brasil, 2018-2019

\begin{tabular}{|c|c|c|c|c|c|c|c|}
\hline VARIÁVEL & $\begin{array}{c}\text { TOTAL } \\
\mathrm{N}=385(\%)\end{array}$ & $\begin{array}{l}\text { CURITIBA } \\
\mathrm{N}=258(\%)\end{array}$ & $\begin{array}{l}\text { SÃO JOSÉ DOS } \\
\text { PINHAIS N=39 (\%) }\end{array}$ & $\begin{array}{c}\text { CAMPO LARGO } \\
\mathrm{N}=23(\%)\end{array}$ & $\begin{array}{l}\text { PINHAIS } \\
\mathrm{N}=28(\%)\end{array}$ & $\begin{array}{c}\text { ARAUCÁRIA } \\
\mathrm{N}=18(\%)\end{array}$ & $\begin{array}{c}\text { ALMIRANTE } \\
\text { TAMANDARÉ } \\
\mathrm{N}=19(\%)\end{array}$ \\
\hline \multicolumn{8}{|c|}{ Identificação do Chamado } \\
\hline Município & $384(99,7)$ & $257(99,6)$ & $39(100)$ & $23(100)$ & $28(100)$ & $18(100)$ & $19(100)$ \\
\hline Data & $385(100)$ & $258(100)$ & $39(100)$ & $23(100)$ & $28(100)$ & $18(100)$ & $19(100)$ \\
\hline Horário & $369(95,8)$ & $234(90,7)$ & $38(97,4)$ & $23(100)$ & $28(100)$ & $18(100)$ & $19(100)$ \\
\hline USA & $385(100)$ & $257(99,6)$ & $39(100)$ & $23(100)$ & $28(100)$ & $18(100)$ & $19(100)$ \\
\hline Base & 381 (99) & $257(99,6)$ & $39(100)$ & $23(100)$ & $25(89,3)$ & $17(94,4)$ & $19(100)$ \\
\hline \multicolumn{8}{|c|}{ Identificação do Paciente } \\
\hline Sexo & $372(96,6)$ & $251(97,3)$ & $38(97,4)$ & $23(100)$ & $27(96,4)$ & $16(88,9)$ & $17(89,5)$ \\
\hline
\end{tabular}




$\begin{array}{lccccccc}\text { Idade } & 375(97,4) & 252(97,7) & 39(100) & 23(100) & 27(96,4) & 17(94,4) & 17(89,5) \\ & 131(34) & 105(40,7) & 13(33,3) & 5(21,7) & 8(28,6) & 3(16,7) & 0(0) \\ \text { Bairro } & 148(38,4) & 110(42,6) & 12(30,8) & 1(4,3) & 10(35,7) & 7(38,9) & 8(42,1) \\ \text { Local de ocorrência } & 148 \text { tificacão demográfica } \\ \text { Fonte: Os autores, 2021. } & & & & & & \end{array}$

Frente as variáveis clínicas do paciente, 30 variáveis coletadas, presente na ficha de ocorrência a completude de preenchimento apresentaram-se excelente para objetivo do chamado (99,7\%), principais sintomas $(98,7 \%)$ e muito ruim para antecedentes
$(42,1 \%)$, cirurgias realizadas $(10,9 \%)$, medicações de uso $(17,4 \%)$, exame clínico geral $(23,1 \%)$, neurológico $(48,6 \%)$ e urogenital $(10,4 \%)$, horário de início dos sintomas $(34 \%)$, temperatura $(33,8 \%)$, escala de trauma $(15,1 \%)$ e achados $(1,8 \%)$. Des- taca-se que Campo Largo foi o município que apresentou o maior número de variáveis com completude de preenchimento muito ruim $(\mathrm{n}=15)$ (Tabela 2$)$.

Entre as variáveis referente ao desfecho do atendimento ( $\mathrm{n}=$ sete), observa-se que

\section{Tabela 2. Completude dos campos de preenchimento das fichas de ocorrência de atendimento das Unidades de Suporte Avançado do SAMU Regional Metropolitano/PR das variáveis clínicas do paciente. Curitiba, Paraná, Brasil, 2018-2019.}

\begin{tabular}{|c|c|c|c|c|c|c|c|}
\hline VARIÁVEL & $\begin{array}{c}\text { TOTAL } \\
\mathrm{N}=385(\%)\end{array}$ & $\begin{array}{l}\text { CURITIBA } \\
\mathrm{N}=258(\%)\end{array}$ & $\begin{array}{c}\text { SÃO JOSÉ } \\
\text { DOS PINHAIS } \\
\text { N=39 (\%) }\end{array}$ & $\begin{array}{l}\text { CAMPO LARGO } \\
\mathrm{N}=23(\%)\end{array}$ & $\begin{array}{l}\text { PINHAIS } \\
\mathrm{N}=28(\%)\end{array}$ & $\begin{array}{c}\text { ARAUCÁRIA } \\
\text { N=18 (\%) }\end{array}$ & $\begin{array}{c}\text { ALMIRANTE } \\
\text { TAMANDARÉ } \\
\mathrm{N}=19(\%)\end{array}$ \\
\hline Objetivo do chamado & $384(99,7)$ & $257(99,6)$ & $39(100)$ & $23(100)$ & $28(100)$ & $18(100)$ & 19 (100) \\
\hline Antecedentes & $162(42,1)$ & $5(1,9)$ & * & $0(0)$ & $2(7,1)$ & $0(0)$ & $0(0)$ \\
\hline Cirurgias realizadas & $42(10,9)$ & $4(1,6)$ & * & $2(8,7)$ & $0(0)$ & $0(0)$ & $0(0)$ \\
\hline Medicações em uso & $67(17,4)$ & $41(15,9)$ & $23(59)$ & $1(4,3)$ & $3(10,7)$ & $0(0)$ & $1(5,3)$ \\
\hline Principais sintomas & $380(98,7)$ & $254(98,4)$ & $39(100)$ & $23(100)$ & $27(96,4)$ & $18(100)$ & $19(100)$ \\
\hline Exame clínico & $89(23,1)$ & $36(14)$ & * & $5(21,7)$ & $5(17,9)$ & $3(16,7)$ & $3(15,8)$ \\
\hline Início dos sintomas & $131(34)$ & $44(17,1)$ & * & $6(26,1)$ & $28(100)$ & $7(38,9)$ & $10(52,6)$ \\
\hline Pressão Arterial (PA) & $341(88,6)$ & $234(90,7)$ & $31(79,5)$ & $20(87)$ & $24(85,7)$ & $16(88,9)$ & $16(84,2)$ \\
\hline Frequência cardíaca (FC) & $362(94)$ & $249(96,5)$ & $31(79,5)$ & $21(91,3)$ & $26(92,9)$ & $17(94,4)$ & $18(94,7)$ \\
\hline Frequência respiratória (FR) & $397(77,1)$ & $192(74,4)$ & $32(82,1)$ & $16(69,6)$ & $26(92,9)$ & $16(88,69)$ & $15(78,9)$ \\
\hline Saturação de 02 & $357(92,7)$ & 245 (95) & $32(82,1)$ & $20(87)$ & $26(92,9)$ & $17(94,4)$ & $17(89,5)$ \\
\hline Temperatura (T) & $130(33,8)$ & $70(27,1)$ & $22(56,4)$ & $10(43,5)$ & $13(46,4)$ & $12(66,7)$ & $5(26,3)$ \\
\hline Escala de Glasgow & $286(74,3)$ & $197(76,4)$ & $28(71,8)$ & $17(73,9)$ & $21(75)$ & $15(83,3)$ & $8(42,1)$ \\
\hline Escala de Trauma & $58(15,1)$ & $28(10,9)$ & $17(43,6)$ & $2(8,7)$ & $5(17,9)$ & $6(33,3)$ & $3(15,8)$ \\
\hline Vias aéreas & $340(88,3)$ & 227 (88) & $35(89,7)$ & $21(91,3)$ & $25(89,3)$ & $15(83,3)$ & $17(89,5)$ \\
\hline Respiração & $349(90,6)$ & $234(90,7)$ & $36(92,3)$ & $21(91,3)$ & $26(92,9)$ & $15(83,3)$ & $17(89,5)$ \\
\hline Ausculta pulmonar & $336(87,3)$ & $227(88)$ & $39(100)$ & $18(78,3)$ & $21(75)$ & $16(88,9)$ & $16(84,2)$ \\
\hline Expansividade & $263(68,3)$ & $166(64,3)$ & 39 (100) & $14(60,9)$ & $15(53,6)$ & $14(77,8)$ & $15(78,9)$ \\
\hline Achados & $7(1,8)$ & $8(3,1)$ & $0(0)$ & $1(4,3)$ & $0(0)$ & $1(5,6)$ & $0(0)$ \\
\hline Circulação corporal & $349(90,6)$ & $237(91,9)$ & $35(89,7)$ & $20(87)$ & $25(89,3)$ & $15(83,3)$ & $17(89,5)$ \\
\hline Edema & $318(82,6)$ & $209(81)$ & 39 (100) & $12(52,2)$ & $0(0)$ & $15(83,3)$ & $15(78,9)$ \\
\hline Perfusão & $300(77,9)$ & $200(77,5)$ & $39(100)$ & $13(56,5)$ & $17(60,7)$ & $15(83,3)$ & $16(84,2)$ \\
\hline Característica do pulso & $297(77,1)$ & $197(76,4)$ & $33(84,6)$ & $15(65,2)$ & $21(75)$ & $14(77,8)$ & $17(89,5)$ \\
\hline Ausculta cardíaca & $248(64,4)$ & $156(60,5)$ & * & $9(39,1)$ & $17(60,7)$ & $13(72,2)$ & $15(78,9)$ \\
\hline $\mathrm{ECG}^{* *}$ & $211(54,8)$ & $137(53,1)$ & * & $5(21,7)$ & $12(42,9)$ & $10(55,6)$ & $8(42,1)$ \\
\hline Exame neurológico & $187(48,6)$ & $116(45)$ & * & $10(43,5)$ & $11(39,3)$ & $4(22,2)$ & $10(52,6)$ \\
\hline
\end{tabular}




\section{artigo}

Ferreira, J.G.; Chevonik, I.E.; Batista, J.; Lenhani, B.R.; Marcondes, L.;

Completude das fichas de ocorrência das unidades de suporte avançado do SAMU

\begin{tabular}{|c|c|c|c|c|c|c|c|}
\hline Exame físico do pescoço & $244(63,4)$ & $164(63,6)$ & * & $6(26,1)$ & $12(42,9)$ & $15(83,3)$ & $8(42,1)$ \\
\hline Exame físico urogenital & $40(10,4)$ & $4(1,6)$ & * & $0(0)$ & $0(0)$ & $0(0)$ & $0(0)$ \\
\hline Exame físico do abdômen & $238(61,8)$ & $150(58,1)$ & * & $6(26,1)$ & $21(75)$ & $15(83,3)$ & $9(47,4)$ \\
\hline
\end{tabular}

a completude é falha frente a cinco variáveis, boa para preenchimento do campo óbito (94\%) e excelente para o campo diagnóstico (95,3\%) (Tabela 3).

\section{DISCUSSÃO}

$\mathrm{Na}$ análise das fichas de ocorrência de atendimento do SAMU Regional Metropolitano/PR, foi evidenciado que à identificação do chamado e identificação do paciente foi excelente, porém quanto a identificação demográfica, que compreende bairro e local de ocorrência, a completude caracterizou-se como muito ruim para todos os municípios. Corroborando com estudo realizado na região sudeste do Brasil, São Paulo ${ }^{9}$, que analisou as fichas dos atendimentos realizados pelo SAMU 192 do município, entre agosto de 2011 e janeiro de 2012, totalizando 2635 fichas, descreve que $33,7 \%$ das fichas tem dados de endereços incompletos e que não possibilitaram a identificação do setor de atendimento, porém o presente estudo apresenta uma porcentagem muito maior frente ao não preenchimento dessas variáveis.

As variáveis referentes à identificação de chamado e demográfica são importantes para os gestores responsáveis realizar o planejamento de ações com a população respeitando suas particularidades. O elevado número de não preenchimento das ocorrências pode ter como justificativa o desconhecimento da importância dessas informações, ou até mesmo a gravidade do paciente que está recebendo o atendimento pelo fato que o profissional não tenha tempo suficiente para que esse preenchimento ocorra de maneira adequada.'

O estudo realizado no nordeste do Brasil, Paraíba ${ }^{10}$, com o objetivo caracterizar as vítimas e suas lesões, a partir dos dados de atendimentos do SAMU, realizado em 2010 com 4.514 atendimentos de vítimas de acidente de trânsito, em 266 (5,9\%) o bairro não foi informado, motivo porque esses eventos não foram incluídos nas análises espaciais. A variável local da ocorrência', informando o logradouro correspondente, apresentou taxa de preenchi- mento inferior a $80 \%$ e, por isso, não foi contemplada no estudo, sinalizando uma completude boa para preenchimento de bairro e regular para local de ocorrência, discordando em números com os dados apresentados por esta pesquisa em que os itens de identificação demográfica teve uma completude muito ruim, independente se o atendimento foi na capital ou região metropolitana.

Podemos observar que no estudo realizado no centro-oeste do Brasil, Mato Grosso $^{11}$, por pesquisa documental, com dados coletados de janeiro de 2012 a dezembro de 2013, na base do SAMU, apresentou uma variação de porcentagem em não preenchimento de fichas de atendimento sistematizado (FAS), o mesmo observado nesse estudo, com falha na completude dos dados. Os autores apontaram que as variáveis onde o preenchimento era realizado no formato de cheklist foram mais preenchidas que as por extenso, o melhor preenchimento foi para os campos "motivo de solicitação", "dados da vítima”, corroborando com este estudo em que apresentou completude excelente

Tabela 3. Completude dos campos de preenchimento das fichas de ocorrência de atendimento das Unidades de Suporte Avançado do SAMU Regional Metropolitano/PR das variáveis segundo desfecho do atendimento. Curitiba, Paraná, Brasil, 2018-2019.

\begin{tabular}{|c|c|c|c|c|c|c|c|}
\hline VARIÁVEL & $\begin{array}{c}\text { TOTAL } \\
\mathrm{N}=386(\%)\end{array}$ & $\begin{array}{l}\text { CURITIBA } \\
\mathrm{N}=258(\%)\end{array}$ & $\begin{array}{c}\text { SÃO JOSÉ } \\
\text { DOS PINHAIS } \\
\text { N=39 (\%) }\end{array}$ & $\begin{array}{l}\text { CAMPO LARGO } \\
\qquad \mathrm{N}=23(\%)\end{array}$ & $\begin{array}{l}\text { PINHAIS } \\
\mathrm{N}=28(\%)\end{array}$ & $\begin{array}{l}\text { ARAUCÁRIA } \\
\mathrm{N}=18(\%)\end{array}$ & $\begin{array}{c}\text { ALMIRANTE } \\
\text { TAMANDARÉ } \\
\mathrm{N}=19(\%)\end{array}$ \\
\hline Diagnóstico & $367(95,3)$ & $253(98,1)$ & $38(97,4)$ & $23(100)$ & $28(100)$ & $14(77,8)$ & $17(89,5)$ \\
\hline Procedimentos realizados & $52(13,5)$ & $44(17,1)$ & $9(23,1)$ & $0(0)$ & $0(0)$ & $0(0)$ & $2(10,5)$ \\
\hline Terapêutica & $192(49,9)$ & $128(49,6)$ & $23(59)$ & $7(30,4)$ & $15(53,6)$ & $11(61,1)$ & $10(52,6)$ \\
\hline Evolução & $243(63,1)$ & $179(69,4)$ & $7(17,9)$ & $17(73,9)$ & $18(64,3)$ & $12(66,7)$ & $13(68,4)$ \\
\hline Encaminhamento & $259(67,3)$ & $194(75,2)$ & $13(33,3)$ & $15(65,2)$ & $16(57,1)$ & $8(44,4)$ & $13(68,4)$ \\
\hline Óbito & 362 (94) & $235(91,1)$ & $39(100)$ & $23(100)$ & $28(100)$ & $18(100)$ & $19(100)$ \\
\hline $\begin{array}{l}\text { Posicionamento de trans- } \\
\text { porte }\end{array}$ & $272(70,6)$ & $181(70,2)$ & * & $15(65,2)$ & $15(53,6)$ & $7(38,9)$ & $16(84,2)$ \\
\hline
\end{tabular}


para objetivo do chamado e identificação da vítima.

Ao que se refere à identificação de paciente, variáveis idade e sexo o presente estudo discorda dos dados apresentados por Fernandes e Tanaka ${ }^{12}$, coleta realizada em outubro de 2012, na Central de Regulação do SAMU no estado de São Paulo com 37.457 ocorrências, destaca baixa completude dos registros de idade e sexo (42,3\%), nesta pesquisa a identificação do paciente apresentou excelente completude.

Em qualquer momento, principalmente no início do atendimento, é de extrema importância saber o histórico do paciente (antecedentes, cirurgias realizadas e medicações de uso), no estudo observamos que esses campos de variáveis não são preenchidos por completo independente do município. Para uma conduta adequada, conhecer o paciente que o profissional está atendendo é essencial para entender a evolução do caso ou até mesmo o que acarretou aquele atendimento emergencial. Nem sempre as informações disponibilizadas pelo paciente são claras e completas, então a realização do exame físico completo aferição de sinais vitais (frequência cardíaca, frequência respiratória, temperatura, saturação de O2, pressão arterial) e anamnese é indispensável no atendimento do paciente. Por isso ressaltamos a importância dessa completude, a qual não foi demonstrada nesta pesquisa, pois somente frequência cardíaca e saturação de $\mathrm{O} 2$ tiveram boa completude, para que o próximo profissional do cuidado consiga dar continuidade de maneira correta sem colocar a vida do paciente e o seu trabalho em risco.

$\mathrm{O}$ estudo mostrou que dentro das sete variáveis de desfecho indicadas, duas tem completude adequada, campo de diagnóstico (excelente) e óbito (boa), tanto em Curitiba quanto na região metropolitana. A completude da variável de óbito deste estudo se assemelha com a pesquisa realizada em Paulo Afonso, na Bahia, Brasil, em que 6,94\% das 331 fichas de ocorrências de atendimento do SAMU referente a acidentes envolvendo motociclistas, não continham registros de óbito. ${ }^{13}$
O diagnóstico ou hipóteses de um provável, responsabilidade este de preenchimento do médico da USA, ajuda a estabelecer qual cuidado ou procedimento deve ser adotado, como forma de melhorar o entendimento e conduta diante das ocorrências. No estudo de Silveira e Taneda ${ }^{11}$, o mesmo relata que as hipóteses diagnósticas foram preenchidas com maior eficiência, com cinco a $6 \%$ das variáveis não estavam sendo preenchidas adequadamente.

As cinco variáveis de desfechos que tiveram completudes não adequadas (<90\%), foram as de procedimentos realizados, terapêutica, evolução, encaminhamento e posição de transporte. Cada um desses itens é indispensável para continuidade do atendimento no serviço subsequente. Dentre eles os procedimentos realizados durante o atendimento, a qual designa o próximo passo a avançar. O registro da terapêutica realizada no atendimento evita incidentes e proporciona uma maior segurança ao paciente, previne incidentes como superdosagens e auxilia na tomada de decisão clínica seguinte.

Referente a evolução na ficha de atendimento, esta completude foi falha, corroborando com o estudo paulista ${ }^{14}$ que destaca a existência da carência de carimbos e falta de assinatura, relato de hora de início e término de atendimento e falta de evolução de enfermagem no âmbito hospitalar, autores ${ }^{13}$ apontam como limitação no seu estudo a falha dos registros de enfermagem e a subnotificação dos dados contidos nas fichas de atendimento do SAMU, estes necessários para a compreensão do atendimento a vítima. Vale destacar que o enfermeiro tem grande responsabilidade sob os registros de enfermagem. A execução do processo de enfermagem (PE) é um dever fundamental, este documento assegura toda a legalidade da assistência prestada pelo enfermeiro e respaldando-o de quaisquer contradições no futuro. Destaca-se que as fichas de ocorrências das USAs do SAMU Regional Metropolitano/PR não possuem campo específico destinado ao registro do $\mathrm{PE}$, os campos são destinados para registro de ambos os profissionais, médicos e enfermeiros.
Dentre as 46 variáveis analisadas nesta pesquisa, quando avaliado o preenchimento de Curitiba e região metropolitana junto, apenas 14 tinham preenchimento adequado (>90\%), com completude boa ou excelente, se igualando com os dados do estudo realizado em Pernambuco, Brasil ${ }^{15}$, que entre 100 fichas de ocorrência do SAMU de Patos, Minas Gerais, Brasil, 95 descumpria pelo menos um dos critérios de preenchimento, necessitando da implementação de um programa de melhoria na qualidade dos registros. Os autores destacam como justificativa de falha da completude das fichas de ocorrência, as não adequadas condições oferecidas ao profissional, como a falta de luminosidade, ambientes insalubres e por se tratar de serviço de urgência e emergência a necessidade de maior agilidade da assistência do paciente sobrando pouco tempo para o preenchimento das informações.

A dificuldade de preenchimento de informações pelos profissionais da saúde também é destacada no estudo realizado no Paraná, corroborando com os dados apresentados, onde a completude dos dados foi excelente somente para os campos obrigatórios do sistema de informação, com isso autores destacam que sistemas específicos facilitam o preenchimento e torna as informações mais fidedignas e completas. ${ }^{16}$

Este estudo destaca como limitação, a realização em seis municípios diferentes, porém todos fazem parte de uma mesma coordenação e região do estado, necessitando ser replicado em outros serviços do Brasil, para análise e comparação de dados.

\section{CONCLUSÃO}

O estudo permitiu evidenciar uma falha do preenchimento das fichas de ocorrência das USA's do SAMU Regional Metropolitano/PR de forma geral, independente da localidade do chamado com completude adequada em apenas 14 variáveis das 46 avaliadas. Curitiba teve uma completude maior que os municípios da região metropolitana nas variáveis de identificação de chamado, paciente e de- 


\section{artigo}

Ferreira, J.G.; Chevonik, I.E.; Batista, J.; Lenhani, B.R.; Marcondes, L.;

Completude das fichas de ocorrência das unidades de suporte avançado do SAMU

mográfica, entretanto para as variáveis clínicas e de desfecho teve maior completude municípios da região metropolitana.

A ficha de ocorrência é o prontuário do paciente no atendimento pré-hospitalar, sendo de extrema importância e necessária para o paciente e para profissional. Garante que os cuidados e segurança do paciente sejam eficientes, a excelente completude garante ao paciente, uma qualidade no atendimento e na continuidade dele gerando respaldo legal ao enfermeiro e cliente. Também auxilia nas pesquisam que contribuem para uma enfermagem mais científica.

Faz-se necessário, portanto, desenvolver mecanismos para estabelecer o preenchimento correto e completo da ficha de ocorrência do SAMU, seja por atividades de capacitação continua demonstrando a importância de tais informações bem como desenvolver um modo mais rápido e fácil de preenchimento. A utilização de aparelhos tecnológicos, como tablet ou celular, com formulários de preenchimento por checklist de campos obrigatórios, poderia ser uma opção.

\section{REFERÊNCIAS}

1. Hanauer MC et al. Caracterização dos atendimentos realizados pelo SAMU. Rev enferm UFPE on line. 2018 [cited 2020 apr. 04]; 12(12):3476-83. Available from: https://periodicos.ufpe.br/ revistas/revistaenfermagem/article/view/231418/30829

2. Ascari RA, Pertille F, Oliveira MCB. A importância dos registros de enfermagem no faturamento hospitalar. Rev enferm UFPE on line. 2018 [cited 2020 apr 4]; 12(6):1717-26. Available from: https://periodicos.ufpe.br/revistas/revistaenfermagem/article/ view/234419/2921.

3. Conselho Federal de Enfermagem (Cofen). Resolução COFEN $n^{\circ}$ 0514/2016. Aprova o Guia de Recomendações para Registros de Enfermagem no Prontuário do Paciente. Conselho Federal de Enfermagem [Internet], Brasília, 05 maio 2006; [cited 2021 apr. 16]. Available from: http://www.cofen.gov.br/resolucao-cofen-no-05142016_41295.html.

4. Silva AGI, Dias BRL. Registro de enfermagem: Uma revisão integrativa da literatura. Rev nursing [online]. 2018 [cited 2021 apr. 16]; 21(246):2476-2481 Available from: http://www.revistanursing.com.br/revistas/247/pg30.pdf.

5. Dantas RAN, Torres GV, Salvetti MG, Dantas DV, Mendonça AEO. Instrumento para avaliação da qualidade da assistência pré-hospitalar móvel de urgência: validação de conteúdo. Rev. Esc. Enferm. USP. 2015; 49(3)381-387. DOI: 10.1590/S0080623420150000300004

6. Lima CRA, Schramm JMA, Coeli CM, Silva MEM. Revisão das dimensões de qualidade dos dados e métodos aplicados na avaliação dos sistemas de informação em saúde. Cad Saúde Publica [online]. 2019 [cited 2021 mar. 28]; 25(10):2095-2109. Available from: http://dx.doi.org/10.1590/S0102-311X2009001000002.

7. Calazans ATS. Qualidade da informação: conceitos e aplicações. Transinformação [online]. 2008 [cited 2020 sep. 28]; 20(1):29-48. Available from: https://www.scielo.br/scielo.php?pi $\mathrm{d}=$ S0103-37862008000100003\&script=sci_abstract\&tlng=pt.

8. Costa JMBS, Frias PG. Avaliação da completitude das variáveis da Declaração de Nascido Vivo de residentes em Pernambuco, Brasil, 1996 a 2005. Cad Saúde Pública [online]. 2009 [cited 2020 sep. 28];25(3): 613-624. Available from: https://doi. org/10.1590/S0102-311X2009000300016

9. Almeida PMV, Dell'Acqua MCQ, Cyrino CMS, Juliani CMCM, Palhares VC, Pavelqueires S. Análise dos atendimentos do SAMU 192: Componente móvel da rede de atenção às urgências e emergências. Esc Anna Nery [online]. 2016 [cited 2020 sep. 28];
20(2):289-295. Available from: https://www.scielo.br/scielo.ph p?pid=S1414-81452016000200289\&script=sci_abstract\&tlng=pt.

10. Soares RAS, Pereira APJT, Moraes RM, Vianna RPT. Caracterização das vítimas de acidentes de trânsito atendidas pelo Serviço de Atendimento Móvel de Urgência (SAMU) no Município de João Pessoa, Estado da Paraíba, Brasil, em 2010. Epidemiol. Serv. Saúde [online]. 2012 [cited 2021 apr. 16]; 21(4):589-600. Available from: http://scielo.iec.gov.br/scielo.php?script=sci_arttext\&pid=S1679-49742012000400008

11. Silveira SC, Taneda M. Análise do preenchimento das fichas de atendimento sistematizado do SAMU-192 realizados pela equipe de enfermagem de Juína/MT. Revista SAjes [online]. 2016 [cited 2021 apr. 16]; 1(1):1-18. Available from: https://www.revista.ajes.edu.br/index.php/sajes/article/view/110.

12. Fernandes FSL, Tanaka OY. Processo de priorização de ocorrências no serviço de atendimento móvel de urgência. Rev baiana enferm [online]. 2018 [cited 2020 oct 20]; 32(e.24463). Available from: https://portalseer.ufba.br/index.php/enfermagem/article/view/24463/15971.

13. Santanna MA, Almeida SM, Souza EN, Silva CJ, Rodrigues JW, Botelho Filho CAL. Perfil das vítimas de acidente motociclístico socorridas pelo Serviço de Atendimento Móvel de Urgência SAMU, no município de Paulo Afonso - Bahia. Revista Saúde e Desenvolvimento. 2019;13(16): 133-147.

14. Dias JVM, Oliveira LG, Moia CMS, Ferreira JS, Silva JHS, Sá de Souza MOL. A percepção do enfermeiro sobre auditoria de enfermagem no âmbito hospitalar. Enferm Bras [online]. 2019 [cited 2021 mar. 16]; 18(6):737-742. Available from: https://www.portalatlanticaeditora.com.br/index.php/enfermagembrasil/article/ view/2853/pdf.

15. Timóteo MSTBA et al. Implantação de ciclo de melhoria nos registros de saúde de serviço pré-hospitalar móvel de urgência. Rev Bras Enferm [online]. 2020 [cited 2021 apr. 16]; 73(4):e.20190049. Available from: https://www.scielo.br/scielo. php?script=sci_arttext\&pid=S0034-71672020000400185

16. Marcondes L, Koller FJ, Sarquis LMM, Mensi C, Consonni D, Kalinke LP. Completude dos dados dos sistemas de informação sobre câncer ocupacional ocasionado pelo amianto. Saúde coletiva [online]. 2019 [cited 2021 apr 15]; 9 (48):1387-1392. Available from: http://www.revistas.mpmcomunicacao.com.br/index. php/saudecoletiva/article/view/100 